\title{
Aneurysm growth and de novo aneurysms during aneurysm surveillance
}

\author{
Joseph C. Serrone, MD, ${ }^{1}$ Ryan D. Tackla, MD, ${ }^{1}$ Yair M. Gozal, MD, PhD,, Dennis J. Hanseman, PhD, ${ }^{1}$ \\ Steven L. Gogela, MD, ${ }^{1}$ Shawn M. Vuong, MD, Jennifer A. Kosty, MD, ${ }^{1}$ Calen A. Steiner, MD, ${ }^{3}$ \\ Bryan M. Krueger, MD, ${ }^{1}$ Aaron W. Grossman, MD, PhD, ${ }^{1}$ and Andrew J. Ringer, MD ${ }^{1,4-6}$
}

\begin{abstract}
1Department of Neurosurgery; ${ }^{2}$ Department of Surgery, Division of Trauma/Critical Care, ${ }^{3}$ University of Cincinnati College of Medicine; and ${ }^{5}$ Department of Radiology, University of Cincinnati College of Medicine; ${ }^{4}$ Comprehensive Stroke Center at the University of Cincinnati Neuroscience Institute; and ${ }^{6}$ Mayfield Clinic, Cincinnati, Ohio
\end{abstract}

\begin{abstract}
OBJECTIVE Many low-risk unruptured intracranial aneurysms (UIAs) are followed for growth with surveillance imaging. Growth of UIAs likely increases the risk of rupture. The incidence and risk factors of UIA growth or de novo aneurysm formation require further research. The authors retrospectively identify risk factors and annual risk for UIA growth or de novo aneurysm formation in an aneurysm surveillance protocol.
\end{abstract}

METHODS Over an 11.5-year period, the authors recommended surveillance imaging to 192 patients with 234 UIAs. The incidence of UIA growth and de novo aneurysm formation was assessed. With logistic regression, risk factors for UIA growth or de novo aneurysm formation and patient compliance with the surveillance protocol was assessed.

RESULTS During 621 patient-years of follow-up, the incidence of aneurysm growth or de novo aneurysm formation was $5.0 \% /$ patient-year. At the 6 -month examination, $5.2 \%$ of patients had aneurysm growth and $4.3 \%$ of aneurysms had grown. Four de novo aneurysms formed (0.64\%/patient-year). Over 793 aneurysm-years of follow-up, the annual risk of aneurysm growth was $3.7 \%$. Only initial aneurysm size predicted aneurysm growth (UIA $<5 \mathrm{~mm}=1.6 \%$ vs $U I A \geq 5 \mathrm{~mm}$ $=8.7 \%, p=0.002)$. Patients with growing UIAs were more likely to also have de novo aneurysms $(p=0.01)$. Patient compliance with this protocol was $65 \%$, with younger age predictive of better compliance $(p=0.01)$.

CONCLUSIONS Observation of low-risk UIAs with surveillance imaging can be implemented safely with good adherence. Aneurysm size is the only predictor of future growth. More frequent (semiannual) surveillance imaging for newly diagnosed UIAs and UIAs $\geq 5 \mathrm{~mm}$ is warranted.

http://thejns.org/doi/abs/10.3171/2015.12.JNS151552

KEY WORDS aneurysm; de novo; growth; subarachnoid hemorrhage; surveillance; vascular disorders

$\mathrm{U}$ NRUPTURED intracranial aneurysms (UIAs) exist in $2 \%$ of the North American population. ${ }^{2}$ The natural history of UIAs has been better defined recently after publication of multiple prospective series. ${ }^{14,16,21,25,28,33,34}$ Results from these observational studies have allowed risk stratification of UIAs to distinguish high-risk UIAs from UIAs with a relatively benign natural history. ${ }^{12}$ With the addition of knowledge from treatment outcomes, we can better weigh the decision to observe or intervene in UIAs.

Most physicians have adopted a conservative approach toward managing small anterior circulation UIAs, which includes observation with surveillance imaging. ${ }^{2,20,36} \mathrm{Re}$ - cent series have shown that aneurysm growth increases the risk of subsequent rupture..$^{13,31}$ This finding has led many physicians to recommend treatment for UIAs with observed growth. The rate of growth, pattern of growth (linear vs episodic), rate of de novo aneurysm formation, and risk factors for growth are inconsistent in the current literature, thus warranting further study in this area. $4,5,7,8,13,17,19,22-24,27-30,32,33$ We report our experience with an aneurysm surveillance protocol in appropriately selected patients and report the incidence and risk factors for growth of UIAs, the incidence of de novo UIA formation, and predictors of compliance to surveillance imaging.

ABBREVIATIONS CTA = CT angiography; DSA = digital subtraction angiography; MRA = MR angiography; SAH = subarachnoid hemorrhage; UIA = unruptured intracranial aneurysm.

SUBMITTED July 3, 2015. ACCEPTED December 1, 2015

INCLUDE WHEN CITING Published online March 11, 2016; DOI: 10.3171/2015.12.JNS151552. 


\section{Methods}

Since July 2001, the senior author has followed with surveillance imaging UIAs felt to be at low risk for spontaneous subarachnoid hemorrhage (SAH) or UIAs whose risks of treatment outweigh the natural history. MR angiography (MRA) is the preferred imaging modality for aneurysm surveillance, which has been validated previously ${ }^{11,26}$ CT angiography (CTA) or rarely digital subtraction angiography (DSA) were performed if contraindications to MRA existed. New images were compared with prior studies by the senior author and a staff radiologist. Only cases with a consensus opinion of growth or morphological change were considered positive. This surveillance protocol included MRA every 6 months for the first 2 years after diagnosis followed by annual MRA for 3 more years to complete 5 years of follow-up. After 5 years of stability, no further imaging was recommended.

Any increase in maximal diameter or a morphological change in the aneurysm was felt to indicate instability in the aneurysm wall, and this finding was usually followed with a recommendation for treatment. To account for delays in diagnosis of aneurysm growth or de novo aneurysm formation encountered with periodic imaging, a corrected time was also calculated as described by others. ${ }^{18}$ The corrected time defined the date of aneurysm growth or de novo aneurysm formation as the date halfway between the imaging with the new finding and the previous imaging study.

To identify patients in aneurysm surveillance, medical records were retrospectively reviewed for patients initially evaluated from July 1, 2001, to December 31, 2012, with follow-up data reviewed through June 30, 2013. This study had approval of the institutional review board. Case collection involved searching for patients seen in clinic with the diagnosis of an untreated UIA and at least 1 followup clinic visit or consultation. This yielded 342 patients. Manual chart review of these cases excluded 150 patients with 1) extradural aneurysms, 2) nonsaccular aneurysms, 3 ) aneurysms that had been treated, and 4) patients with fewer than 2 imaging studies. We identified 192 patients in aneurysm surveillance for further analysis.

Chart review of these patients involved documentation of reason for initial imaging, aneurysm location, aneurysm size, de novo aneurysms, sex, age at diagnosis, aspirin use, hypertension, tobacco smoking status (ever used tobacco or never used tobacco), history of cerebrovascular disease, family history of SAH, personal history of SAH, ethanol use, and illicit drug use. Since patients with a history of SAH were usually offered treatment instead of surveillance, only 2 surveilled patients had a history of SAH. Thus, statistical analysis was not performed for this risk factor. The aneurysm location was classified into internal carotid artery, posterior communicating artery, anterior communicating artery/anterior cerebral artery, middle cerebral artery, or "posterior" for vertebrobasilar and posterior cerebral artery aneurysms.

Final outcomes of surveillance were recorded. This included whether a patient remained compliant with the surveillance protocol. Medical records were reviewed for evidence of spontaneous SAH. If any treatment occurred, the type of treatment, angiographic result, and clinical outcome were documented.

\section{Statistical Analysis}

Logistic regression analyses for the 192 patients and 234 aneurysms were done separately with select explanatory variables for aneurysm growth or de novo aneurysm formation. Univariate logistic regression was performed to identify variables predicting aneurysm growth or de novo aneurysm formation for the following (values in parentheses represent the number of patients with missing data): age, sex, imaging reason, aspirin use (6), hypertension (6), tobacco use (6), history of cerebrovascular disease (8), family history of SAH (7), ethanol use (6), and illicit drug use (6). To identify aneurysm-specific variables predicting growth, univariate logistic regression was performed for the following (no missing data): aneurysms size, aneurysm location, and multiplicity. To identify variables predicting patient compliance to the aneurysm surveillance protocol, univariate logistic regression was performed for the following (missing data): age, sex, family history of SAH (7), tobacco use (6), ethanol use (6), and illicit drug use (6). No model had multiple explanatory variables with $\mathrm{p}$ values $<0.20$ in univariate analysis, so multivariate logistic regression analysis was not performed. Fisher's exact test was used to determine any association between patients with growth of UIAs and de novo aneurysm formation. Kaplan-Meier curves are presented for time to aneurysm growth as well as for time to either growth or de novo aneurysm formation (Figs. 1 and 2, respectively). Because our logistic regression analysis identified aneurysm size as a risk factor for growth, we performed a Kaplan-Meier analysis after stratifying aneurysms by size $(<5 \mathrm{~mm}$ and $\geq 5 \mathrm{~mm}$ ) (Fig. 3).

Odds ratios with $95 \%$ confidence intervals were calculated and are given in Table 1 for aneurysm growth; $\mathrm{p}$ values $<0.05$ were considered significant. IBM SPSS Statistics (version 20) and SAS (version 9.3) software were used for statistical analysis.

\section{Literature Search and Study Eligibility}

We searched the PubMed database on August 1, 2014. The following search was performed: (cerebral OR intracranial) AND aneurysm AND (growth OR "de novo").

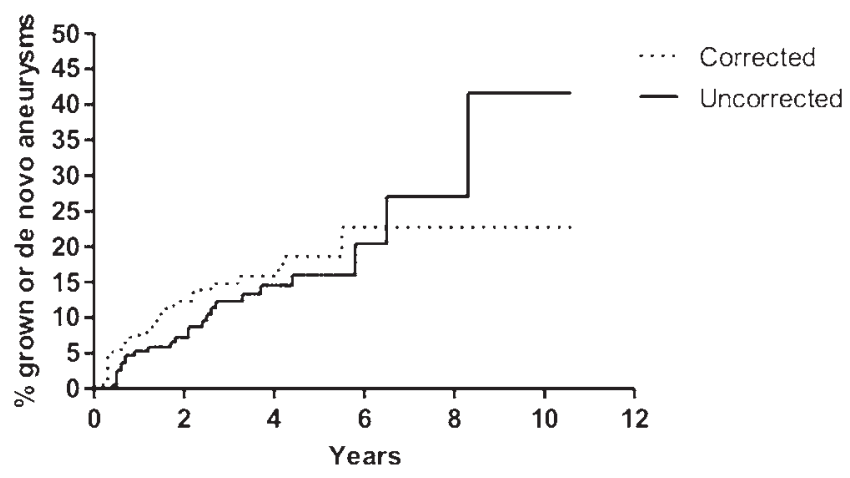

FIG. 1. Kaplan-Meier curve showing the percentage of patients with a growing aneurysm or de novo aneurysm formation. The solid line is the uncorrected time until growth of an aneurysm or de novo aneurysm formation and the dotted line is the corrected time until growth of an aneurysm or de novo aneurysm formation. 


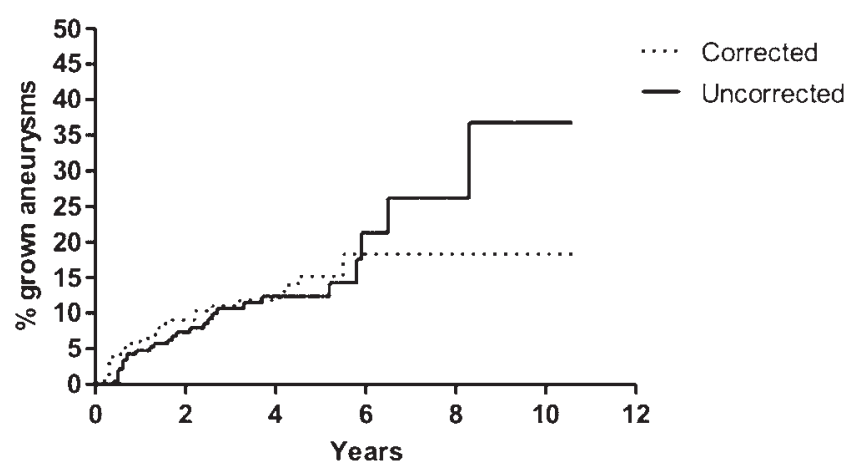

FIG. 2. Kaplan-Meier curve showing the percentage of aneurysms with growth over time. The solid line is the uncorrected time until aneurysm growth and the dotted line is the corrected time until aneurysm growth.

For the aneurysm growth literature review, retrospective or prospective series with at least 50 patients reporting patient number, aneurysm number, duration of follow-up, and number of growing aneurysms were included. The de novo aneurysm formation literature review included all retrospective or prospective series with at least 50 patients reporting number of patients, duration of follow-up, and number of de novo aneurysms. For series from the same institutions overlapping the same time period, the largest series were chosen. Titles and abstracts were reviewed to identify relevant articles. Reference lists of relevant studies were screened for additional relevant studies.

\section{Results}

We evaluated 192 patients with 234 UIAs in our surveillance protocol. Baseline characteristics are presented in Table 1. Excluding the initial imaging, there were 746 surveillance imaging studies with a mean of 3.9 studies per patient. The initial imaging modality was MRA in $73 \%$, CTA in $20 \%$, and DSA in $7 \%$. Subsequent studies were MRA in $87 \%$, CTA in $9 \%$, and DSA in $4 \%$. There was an average follow-up duration of 3.2 years per patient and 3.3 years per aneurysm, yielding 621 patient-years of follow-up and 793 aneurysm-years of follow-up. Seventyone percent of patients had at least 2 years of imaging follow-up.

\section{Overall Surveillance Results}

During the surveillance period, growth was identified in 28 of 234 UIAs followed in 192 patients. Additionally, 4 de novo aneurysms formed over the same period. In 31 of 746 surveillance imaging studies, a growing aneurysm and/or de novo aneurysm was discovered (27 studies with growing aneurysms, 3 studies with de novo aneurysms, and 1 study with a growing aneurysm and a de novo aneurysm). These 31 events occurring during 621 patient-years of follow-up led to a 5.0\% incidence of aneurysm growth or de novo aneurysm formation per patient-year of followup. Growth or de novo aneurysm formation at the 6-month examination was found in 10 patients (5.2\%). The KaplanMeier curve in Fig. 1 illustrates the percentage of patients with a growing aneurysm or de novo aneurysm over time. The mean uncorrected time until growth or de novo aneu-

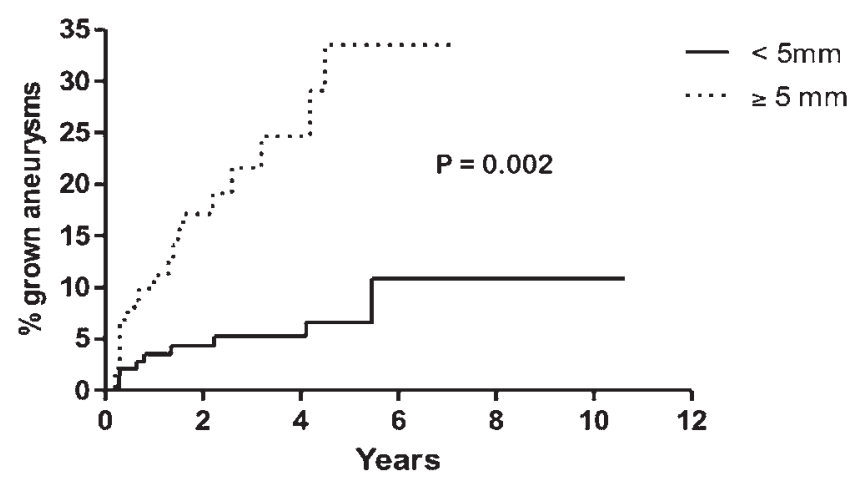

FIG. 3. Kaplan-Meier curve showing the percentage of aneurysms with growth over time dichotomized by size. Both lines are the corrected time until growth.

rysm formation was 2.2 years (range $0.4-5.8$ years) with a corrected time of 1.4 years (range $0.2-5.5$ years).

\section{Aneurysm Growth}

The incidence of aneurysm growth per aneurysm-year of follow-up was $3.7 \%$. No growth was seen in 206 aneurysms (88\%), 10 aneurysms (4.3\%) had early growth at the initial 6-month examination, 17 aneurysms (7.3\%) had late growth, and 1 aneurysm grew at a late examination but did not have early imaging to rule out early growth. Figure 2 illustrates the percentage of aneurysms with growth over time through Kaplan-Meier curves. The mean uncorrected time until aneurysm growth was 2.4 years (range $0.4-8.3$ years) and the corrected time was 1.5 years (range $0.25-5.5$ years) (Fig. 2). An enlargement in the maximum aneurysm diameter was seen in 25 aneurysms and a morphological change was seen in 3 aneurysms. The mean growth was $1.9 \mathrm{~mm}$ (range $0.7-6 \mathrm{~mm}$ ).

Initial aneurysm size was the only predictor of aneurysm growth $(\mathrm{p}=0.002)$ (Table 1$)$. The OR for continuous aneurysm size predicting aneurysm growth was 1.15 (95\% CI 1.05-1.27) so that every 1-mm increase of initial aneurysm diameter increased the risk of growth during the protocol by $15 \%$.The incidence of growth for UIAs < $5 \mathrm{~mm}$ versus UIA $\geq 5 \mathrm{~mm}$ was $1.6 \%$ and $8.7 \%$ per aneurysm-year, respectively $(\mathrm{p}=0.002)$ (Fig. 3).

\section{De Novo Aneurysm Formation}

Four of 192 patients developed de novo aneurysms during follow-up at an average uncorrected time until formation of 1.4 years (range 0.7-2.1 years) with a corrected time of 1.0 years (range $0.3-1.8$ years). The incidence of de novo aneurysm formation per patient-year of follow-up was $0.64 \%$. No patient-specific factor predicted de novo aneurysm formation. However, 3 of 4 patients with de novo aneurysms also had a growing aneurysm, and this was statistically significant (Fisher's exact, $\mathrm{p}=0.01$ ).

\section{Protocol Compliance, Treatment Outcomes, and Occurrence of SAH}

Compliance with surveillance imaging was seen in $65 \%$ of patients. Older patients were less likely to be compliant with each additional year of age, decreasing compli- 
TABLE 1. Baseline characteristics and risk factors for aneurysm growth and de novo aneurysm formation in 192 patients with 234 aneurysms*

\begin{tabular}{|c|c|c|c|c|c|c|}
\hline $\begin{array}{c}\text { Baseline Characteristic/ } \\
\text { Risk Factor }\end{array}$ & Number & $\begin{array}{l}\text { Growing or De Novo } \\
\text { Aneurysm (\%) }\end{array}$ & $\begin{array}{l}\text { No Growing or De } \\
\text { Novo Aneurysm (\%) }\end{array}$ & OR & $95 \% \mathrm{Cl}$ & p Value \\
\hline \multicolumn{7}{|l|}{ Patient-based data } \\
\hline Number of patients & 192 & $29(14.6)$ & $163(85.4)$ & & & \\
\hline Sex & & & & 1.03 & $0.35-3.02$ & 0.81 \\
\hline Male & 40 & $6(12.5)$ & $34(87.5)$ & & & \\
\hline Female & 152 & $22(14.5)$ & $130(85.5)$ & & & \\
\hline Mean age in yrs \pm SD & $61.1 \pm 12.5$ & $62.1 \pm 12.3$ & $60.9 \pm 12.6$ & 1.008 & $0.98-1.04$ & 0.53 \\
\hline \multicolumn{7}{|l|}{ Imaging reason } \\
\hline Stroke/TIA & 39 & $4(10.6)$ & $35(89.4)$ & Reference & Reference & Reference† \\
\hline Screening & 11 & $2(18.1)$ & $9(81.9)$ & 1.51 & $0.25-9.11$ & 0.79 \\
\hline Headache & 62 & $9(14.5)$ & $53(85.5)$ & 1.16 & $0.36-3.74$ & 0.84 \\
\hline Dizziness & 24 & $3(12.5)$ & $21(87.5)$ & 0.97 & $0.21-4.49$ & 0.67 \\
\hline Memory loss & 9 & $2(22.2)$ & $7(78.8)$ & 1.94 & $0.31-12.12$ & 0.54 \\
\hline Vision loss & 8 & $1(12.5)$ & $7(87.5)$ & 0.97 & $0.098-9.65$ & 0.79 \\
\hline Other/unknown & 39 & $7(17.9)$ & $32(82.1)$ & 1.49 & $0.43-5.17$ & 0.69 \\
\hline Aspirin & & & & 0.72 & $0.29-1.81$ & 0.17 \\
\hline Yes & 75 & $8(10.7)$ & $67(90.3)$ & & & \\
\hline No & 111 & $20(18.0)$ & $91(82.0)$ & & & \\
\hline HTN & & & & 0.77 & $0.33-1.82$ & 0.80 \\
\hline Yes & 107 & $16(15.0)$ & $91(85.0)$ & & & \\
\hline No & 79 & $11(13.9)$ & $68(86.1)$ & & & \\
\hline Tobacco & & & & 2.02 & $0.81-5.00$ & 0.26 \\
\hline Ever used & 115 & $20(17.4)$ & $95(82.6)$ & & & \\
\hline Never used & 71 & $7(9.8)^{\prime}$ & 64 (90.2) & & & \\
\hline CVD & & & & 0.43 & $0.13-1.45$ & 0.57 \\
\hline Yes & 38 & $3(7.9)$ & 35 (92.1) & & & \\
\hline No & 146 & $24(16.4)$ & $122(83.6)$ & & & \\
\hline Family $\mathrm{Hx}$ of $\mathrm{SAH}$ & & & & 1.06 & $0.29--3.85$ & 0.96 \\
\hline Yes & 20 & $3(15)$ & $17(85)$ & & & \\
\hline No & 165 & $24(14.5)$ & $141(85.5)$ & & & \\
\hline EtOH use & & & & 0.54 & $0.21-1.38$ & 0.52 \\
\hline Yes & 58 & $7(12.1)$ & $51(87.9)$ & & & \\
\hline No & 128 & $20(15.6)$ & $108(84.4)$ & & & \\
\hline Illicit drug use & & & & 0.16 & $0.01-2.58$ & 0.24 \\
\hline Yes & 15 & $0(0)$ & $15(100)$ & & & \\
\hline No & 171 & $27(15.8)$ & $144(84.2)$ & & & \\
\hline \multicolumn{7}{|l|}{ Aneurysm-based data } \\
\hline Number of aneurysms & 234 & $28(12.0)$ & $206(88.0)$ & & & \\
\hline \multicolumn{7}{|l|}{ Location } \\
\hline ACoA/ACA & $44(18.9)$ & $3(6.8)$ & $41(93.2)$ & Reference & Reference & Referenceł \\
\hline ICA & $80(34.2)$ & $11(13.8)$ & $69(86.2)$ & 2.47 & $0.60-10.1$ & 0.21 \\
\hline PCoA & $21(9.0)$ & $3(14.3)$ & $18(85.7)$ & 2.67 & $0.46-15.4$ & 0.27 \\
\hline MCA & $67(28.6)$ & $7(10.4)$ & $60(89.6)$ & 1.38 & $0.30-6.30$ & 0.68 \\
\hline Posterior§ & $22(9.4)$ & $4(18.1)$ & $18(81.9)$ & 2.73 & $0.50-14.8$ & 0.25 \\
\hline Aneurysm size & & & & 1.15 & $1.05-1.27$ & $0.0024 \pi$ \\
\hline$<3 \mathrm{~mm}$ & $48(20.5)$ & $1(2.1)$ & 37 (97.9) & & & \\
\hline $3-6 \mathrm{~mm}$ & $146(62.4)$ & $18(12.3)$ & $128(87.7)$ & & & \\
\hline $7-11 \mathrm{~mm}$ & $32(13.7)$ & $8(25.0)$ & $24(75.0)$ & & & \\
\hline $12-24 \mathrm{~mm}$ & $8(3.4)$ & $1(12.5)$ & $7(87.5)$ & & & \\
\hline$>25 \mathrm{~mm}$ & 0 & & & & & \\
\hline
\end{tabular}


» CONTINUED FROM PAGE 1377

TABLE 1. Baseline characteristics and risk factors for aneurysm growth and de novo aneurysm formation in 192 patients with 234 aneurysms*

\begin{tabular}{lccccc}
\hline $\begin{array}{c}\text { Baseline Characteristicl } \\
\text { Risk Factor }\end{array}$ & Number & $\begin{array}{c}\text { Growing or De Novo } \\
\text { Aneurysm (\%) }\end{array}$ & $\begin{array}{c}\text { No Growing or De } \\
\text { Novo Aneurysm (\%) }\end{array}$ & OR & $95 \% \mathrm{Cl}$ \\
\hline $\begin{array}{l}\text { Aneurysm-based data (continued) } \\
\text { Multiplicity } \\
\text { Yes }\end{array}$ & & & & \\
No & $73(31.2)$ & $8(11.0)$ & $65(89.0)$ & 0.87 & $0.35-2.20$ \\
\hline
\end{tabular}

$\mathrm{ACA}=$ anterior cerebral artery; $\mathrm{ACOA}=$ anterior communicating artery; $\mathrm{CVD}=$ cerebrovascular disease; $\mathrm{EtOH}=\mathrm{ethanol}$; $\mathrm{HTN}=$ hypertension; $\mathrm{Hx}=$ history; $\mathrm{ICA}=$ internal carotid artery; $\mathrm{MCA}=$ middle cerebral artery; $\mathrm{PCOA}=$ posterior communicating artery; TIA = transient ischemic attack.

* Results of univariate logistic regression analysis are presented. Given that neither the patient-based data nor aneurysm-based data had 2 variables with a $p$ value < 0.20 , multivariate analysis was not performed for these models.

$\dagger$ All statistical comparisons were made to stroke/transient ischemic attack, which had the lowest incidence of growth.

$\ddagger$ All statistical comparisons were made to anterior communicating artery/anterior cerebral artery, which had the lowest incidence of growth.

$\S$ Indicates vertebrobasilar and posterior cerebral artery aneurysms.

If $p$ value calculated from continuous data from aneurysm size.

ance by $3 \%$ (OR 0.97 [95\% CI 0.94-0.99], $\mathrm{p}=0.01$ ). Treatment was undertaken in 22 of 32 growing or de novo aneurysms over 621 patient-years of follow-up (3.5\% chance of treatment per patient-year of follow-up). Ten growing or de novo aneurysms were not treated because 4 patients declined treatment, 3 patients were of advanced age, 1 patient was too medically ill, 1 de novo aneurysm was deemed too small for treatment, and 1 de novo aneurysm was observed because the initial surveillance aneurysm was a stable basilar terminus aneurysm of higher risk.

Of 22 patients receiving treatment, 7 underwent clip ligation and 15 received endovascular treatment. Twenty patients had a good neurological outcome with complete or near-complete aneurysm occlusion. One patient had partial monocular vision loss after embolization of an ophthalmic segment ICA aneurysm, and another patient died of a retroperitoneal hematoma after placement of a stent for staged treatment of a growing basilar terminus aneurysm.

Aneurysmal SAH occurred during follow-up in 1 patient and after completion of follow-up in another patient. The first patient was an 85-year-old man with a $14-\mathrm{mm}$ basilar terminus aneurysm that was stable for 1.2 years into surveillance. He became noncompliant with imaging surveillance and presented 2.3 years after initial diagnosis with aneurysmal SAH. The other patient was a 66-yearold woman with a $10-\mathrm{mm}$ basilar aneurysm that was stable on imaging for 4.7 years. She presented with SAH 3.5 years after completion of follow-up. Of note, both aneurysms were larger on their treatment DSA studies than on prior images.

\section{Discussion}

This report demonstrates a high rate of early growth in surveilled UIAs and much higher rate of growth in UIAs $\geq 5 \mathrm{~mm}$. The study also demonstrates the feasibility of implementing an aneurysm surveillance protocol into practice for appropriately selected UIAs. The high rate of early growth in surveilled UIAs implicates that these lesions should be imaged more frequently during the initial surveillance period. Second, due to an increased incidence of growth for aneurysms $\geq 5 \mathrm{~mm}$ in diameter, these UIAs may warrant more frequent surveillance imaging, longer duration surveillance, or immediate occlusion. With these considerations, any recommendation regarding imaging frequency must balance costs and patient inconvenience with the risk of SAH due to an undetected growing UIA.

The concept of a growing aneurysm being unstable and posing a risk of imminent SAH has support from recent publications. These so-called "Yonekura type 2 " aneurysms, are defined by growth immediately prior to rupture. ${ }^{35}$ Inoue et al. found growing aneurysms that subsequently underwent surgical clipping to have walls that were "reddish, thin, and fragile" intraoperatively. ${ }^{13}$ Frösen et al. have noticed similar thin hypocellular aneurysm walls (Type D) to be most common in ruptured aneurysms. ${ }^{10}$ These same investigators suggest that loss of smooth muscle cells in the aneurysm wall underlies growth and subsequent rupture. ${ }^{9}$

The incidence of rupture from an acutely growing untreated UIA has been evaluated in 2 series in the literature. Inoue et al. cited an annual incidence of rupture in growing UIAs at $18.5 \%$. Because some growing aneurysms were treated in their study (i.e., interrupting the natural history), the true risk of hemorrhage could be as high as $45 \%$. Due to their use of low-resolution MRA, these investigators subselected very significantly enlarging UIAs. This accounted for the lower overall annual risk of growth $(1.8 \%)$ with a very high annual incidence of rupture among growing UIAs (18.5\%). ${ }^{13}$

Villablanca et al. followed UIAs with CTA, a more sensitive methodology to detect growth. This highly sensitive method underlies their very high reported annual growth rate of $18 \%$. Because they selected very subtly growing aneurysms, they found a lower annual risk of rupture of growing UIAs (2.4\%). However, Villablanca et al. similarly found growing UIAs to be at higher risk of SAH. In their series, the risk of rupture in growing UIAs was 12fold higher than the risk of rupture in nongrowing UIAs ( $2.4 \%$ vs $0.2 \%, \mathrm{p}=0.034) .{ }^{31}$ These 2 studies highlight how the incidence of UIA growth or the incidence of rupture in 


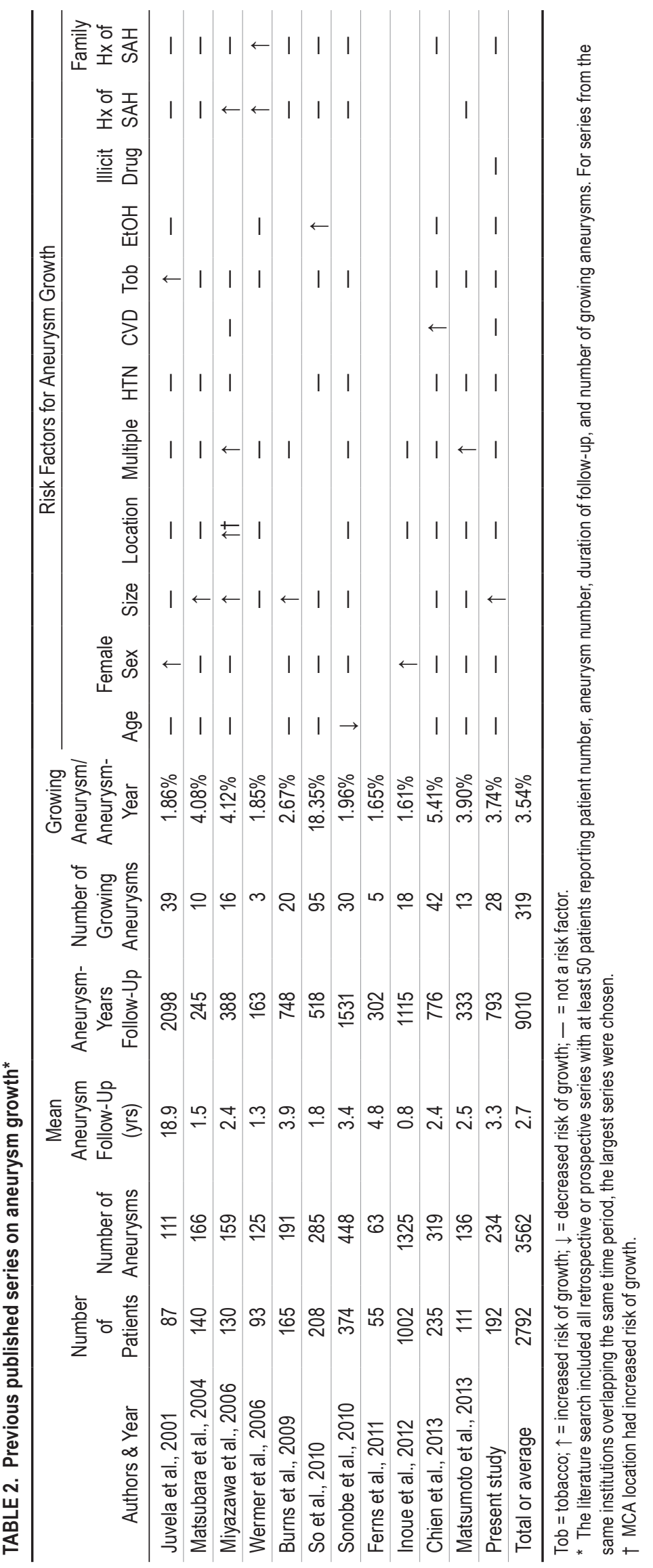


growing UIAs can depend on the sensitivity of the imaging modality. More importantly, they strongly suggest that growing UIAs are unstable and are at higher risk for $\mathrm{SAH}$ than nongrowing UIAs.

Based on the premise that growing UIAs are at higher risk of rupture, assessment of risk factors for aneurysm growth, especially modifiable risk factors, is clinically pertinent. The annual incidence of UIA growth is about $3.5 \%$ and the annual incidence of de novo aneurysm formation approaches $1 \%$ (Tables 2 and 3). As is evident from Table 2, aneurysm size is the most consistent risk factor for aneurysm growth from the literature. We found larger aneurysm size to be the only predictor of aneurysm growth. Of 9 previous studies evaluating aneurysm size as a risk factor for aneurysm growth, 3 found that larger aneurysms grow more than smaller aneurysms (Table 2). These studies reported that UIAs larger than $5 \mathrm{~mm}, 8 \mathrm{~mm}$, and 10 $\mathrm{mm}$ were more likely to grow. ${ }^{4,22,24}$ We noted an increased risk of growth for aneurysms that are $\geq 5 \mathrm{~mm}$ (Fig. 3). Since the risk of aneurysm rupture begins to increase at $7 \mathrm{~mm}, 5 \mathrm{~mm}$ may serve as a reasonable size to increase surveillance intensity or offer immediate treatment for aneurysm occlusion.

The ideal frequency of surveillance imaging for UIAs is not currently well defined. ${ }^{3}$ Long-term frequent surveillance imaging is costly and burdensome to patients. However, the growth of aneurysms is likely nonlinear with episodic phases of growth being associated with high risks of SAH. ${ }^{1,6,15,19}$ We found the rate of growth at the 6-month examination to be much higher than expected. Using the multiplicative law of probability with an annual growth rate of $3.74 \%$, and the occurrence of 6-month examination at 0.56 years, we find the expected incidence of aneurysm growth for a linear model would be $2.11 \%$ :

$$
\begin{aligned}
& \text { Annual Risk of Growth }=3.74 \%=0.0374 \\
& \text { Annual Risk of No Growth }=1-0.0374=0.9626 \\
& \text { Risk of Growth }=1-(\text { Annual Risk of No } \\
& \text { Growth })^{\text {follow-up period in years }} \\
& 2.11 \%=1-(0.9626)^{0.56}
\end{aligned}
$$

However, $4.3 \%$ of aneurysms in our series grew as noted on the 6-month examination, suggesting more frequent early imaging is necessary to identify acutely growing UIAss for intervention to prevent SAH. Inoue et al. predicted that the likelihood of finding a growing aneurysm before rupture was $81.5 \%$ if evaluated every 12 months and $90.3 \%$ if evaluated every 6 months. ${ }^{13}$ Given this much improved sensitivity with semiannual examinations and the higher than expected incidence of aneurysm growth seen at the 6-month examination in our protocol, it is our contention that future guidelines should recommend more frequent early surveillance imaging.

Modern treatment algorithms are beginning to define the natural history of specific aneurysms by the aneurysm location, aneurysm size, patient history of SAH, hypertension, and genetic risk factors. ${ }^{12}$ New guidelines should also include documented growth during surveillance as a risk factor for aneurysm rupture. As more data are gained about the growth and formation of aneurysms, guidelines may begin to recommend treatment in younger patients if predictive modeling suggests an aneurysm is likely to grow and require treatment within a patient's lifetime. This model of treatment may save costs by foregoing years of unnecessary surveillance imaging for aneurysms that will be treated anyway, reduce morbidity by offering treatment to patients while they are younger with smaller aneurysms (both predictive of better treatment outcomes), and, most importantly, reduce the incidence of SAH. ${ }^{34}$

Our study has several limitations. One limitation of this study is its retrospective design. Although the surveillance protocol was initiated in a prospective manner by the senior author, case selection and data collection were performed retrospectively. The imaging modalities and protocols used were nonuniform as was the interpretation of the new imaging studies by multiple radiologists. Long-term follow-up after completion of surveillance was not performed, meaning that some patients could have had delayed SAH undetected in this study. Lastly, almost the entire study population has no history of SAH and this should be acknowledged with comparison with other patient populations.

TABLE 3. Previously published series on de novo aneurysm formation*

\begin{tabular}{lccccc}
\hline \multicolumn{1}{c}{ Authors \& Year } & $\begin{array}{c}\text { Number of } \\
\text { Patients }\end{array}$ & $\begin{array}{c}\text { Mean Patient } \\
\text { Follow-Up (yrs) }\end{array}$ & $\begin{array}{c}\text { Number of Patients w/ } \\
\text { De Novo Aneurysms }\end{array}$ & $\begin{array}{c}\text { Patient-Years } \\
\text { Follow-Up }\end{array}$ & $\begin{array}{c}\text { De Novo Aneurysm/ } \\
\text { Patient-Year }\end{array}$ \\
\hline David et al., 1999 & 102 & 4.3 & 6 & 443 & $1.35 \%$ \\
\hline Juvela et al., 2001 & 87 & 18.9 & 15 & 1644 & $0.91 \%$ \\
\hline Tsutsumi et al., 2001 & 112 & 9.3 & 9 & 1042 & $0.86 \%$ \\
\hline Wermer et al., 2005 & 610 & 8.3 & 61 & 5078 & $1.20 \%$ \\
\hline Miyazawa et al., 2006 & 130 & 2.4 & 3 & 317 & $0.95 \%$ \\
\hline Sprengers et al., 2009 & 65 & 5.1 & 1 & 1325 & $0.30 \%$ \\
\hline Ferns et al., 2011 & 276 & 4.8 & 2 & 272 & $0.15 \%$ \\
\hline Matsumoto et al., 2013 & 111 & 2.5 & 2 & 621 & $0.74 \%$ \\
\hline Present study & 192 & 3.2 & 4 & 11074 & $0.64 \%$ \\
\hline Total or average & 1685 & 6.6 & 103 & $0.93 \%$ \\
\hline * The literature search included all retrospective or prospective series with at least 50 patients reporting patient number, duration of follow-up, \\
and number of de novo aneurysms.
\end{tabular}




\section{Conclusions}

The annual risk of growth for UIAs is approximately $3.5 \%$ and the risk of de novo aneurysm formation during surveillance of UIAs approaches $1 \%$. The risk of aneurysm growth is highest in the early period of surveillance and is increased for larger aneurysms ( $\geq 5 \mathrm{~mm}$ ). Future guidelines on the management of UIAs should recommend an increased frequency of early surveillance imaging. More frequent imaging or immediate treatment for aneurysms $\geq$ $5 \mathrm{~mm}$ should be considered.

\section{Acknowledgments}

We acknowledge the significant contribution of Eric Kappes in information systems at the Mayfield Clinic for data acquisition.

\section{References}

1. Allcock JM, Canham PB: Angiographic study of the growth of intracranial aneurysms. J Neurosurg 45:617-621, 1976

2. Brown RD: Unruptured intracranial aneurysms. Semin Neurol 30:537-544, 2010

3. Brown RD Jr, Broderick JP: Unruptured intracranial aneurysms: epidemiology, natural history, management options, and familial screening. Lancet Neurol 13:393-404, 2014

4. Burns JD, Huston J III, Layton KF, Piepgras DG, Brown RD $\mathrm{Jr}$ : Intracranial aneurysm enlargement on serial magnetic resonance angiography: frequency and risk factors. Stroke 40:406-411, 2009

5. Chien A, Liang F, Sayre J, Salamon N, Villablanca P, Viñuela F: Enlargement of small, asymptomatic, unruptured intracranial aneurysms in patients with no history of subarachnoid hemorrhage: the different factors related to the growth of single and multiple aneurysms. J Neurosurg 119:190-197, 2013

6. Chmayssani M, Rebeiz JG, Rebeiz TJ, Batjer HH, Bendok BR: Relationship of growth to aneurysm rupture in asymptomatic aneurysms $\leq 7 \mathrm{~mm}$ : a systematic analysis of the literature. Neurosurgery 68:1164-1171, 2011

7. David CA, Vishteh AG, Spetzler RF, Lemole M, Lawton MT, Partovi S: Late angiographic follow-up review of surgically treated aneurysms. J Neurosurg 91:396-401, 1999

8. Ferns SP, Sprengers ME, van Rooij WJ, van den Berg R, Velthuis BK, de Kort GA, et al: De novo aneurysm formation and growth of untreated aneurysms: a 5-year MRA followup in a large cohort of patients with coiled aneurysms and review of the literature. Stroke 42:313-318, 2011

9. Frösen J: Smooth muscle cells and the formation, degeneration, and rupture of saccular intracranial aneurysm wall-a review of current pathophysiological knowledge. Transl Stroke Res 5:347-356, 2014

10. Frösen J, Piippo A, Paetau A, Kangasniemi M, Niemelä M, Hernesniemi J, et al: Remodeling of saccular cerebral artery aneurysm wall is associated with rupture: histological analysis of 24 unruptured and 42 ruptured cases. Stroke 35:2287-2293, 2004

11. Grandin CB, Mathurin P, Duprez T, Stroobandt G, Hammer F, Goffette P, et al: Diagnosis of intracranial aneurysms: accuracy of MR angiography at $0.5 \mathrm{~T}$. AJNR Am J Neuroradiol 19:245-252, 1998

12. Greving JP, Wermer MJ, Brown RD Jr, Morita A, Juvela S, Yonekura M, et al: Development of the PHASES score for prediction of risk of rupture of intracranial aneurysms: a pooled analysis of six prospective cohort studies. Lancet Neurol 13:59-66, 2014

13. Inoue T, Shimizu H, Fujimura M, Saito A, Tominaga
T: Annual rupture risk of growing unruptured cerebral aneurysms detected by magnetic resonance angiography. $\mathbf{J}$ Neurosurg 117:20-25, 2012

14. Ishibashi T, Murayama Y, Urashima M, Saguchi T, Ebara M, Arakawa H, et al: Unruptured intracranial aneurysms: incidence of rupture and risk factors. Stroke 40:313-316, 2009

15. Juvela S, Porras M, Heiskanen O: Natural history of unruptured intracranial aneurysms: a long-term follow-up study. J Neurosurg 79:174-182, 1993

16. Juvela S, Poussa K, Lehto H, Porras M: Natural history of unruptured intracranial aneurysms: a long-term follow-up study. Stroke 44:2414-2421, 2013

17. Juvela S, Poussa K, Porras M: Factors affecting formation and growth of intracranial aneurysms: a long-term follow-up study. Stroke 32:485-491, 2001

18. Kano H, Kondziolka D, Flickinger JC, Yang HC, Flannery TJ, Niranjan A, et al: Stereotactic radiosurgery for arteriovenous malformations, Part 4: management of basal ganglia and thalamus arteriovenous malformations. J Neurosurg 116:33-43, 2012

19. Koffijberg H, Buskens E, Algra A, Wermer MJ, Rinkel GJ: Growth rates of intracranial aneurysms: exploring constancy. J Neurosurg 109:176-185, 2008

20. Komotar RJ, Mocco J, Solomon RA: Guidelines for the surgical treatment of unruptured intracranial aneurysms: the first annual J. Lawrence pool memorial research symposium-controversies in the management of cerebral aneurysms. Neurosurgery 62:183-194, 2008

21. Lee EJ, Lee HJ, Hyun MK, Choi JE, Kim JH, Lee NR, et al: Rupture rate for patients with untreated unruptured intracranial aneurysms in South Korea during 2006-2009. J Neurosurg 117:53-59, 2012

22. Matsubara S, Hadeishi H, Suzuki A, Yasui N, Nishimura $\mathrm{H}$ : Incidence and risk factors for the growth of unruptured cerebral aneurysms: observation using serial computerized tomography angiography. J Neurosurg 101:908-914, 2004

23. Matsumoto K, Oshino S, Sasaki M, Tsuruzono K, Taketsuna $\mathrm{S}$, Yoshimine T: Incidence of growth and rupture of unruptured intracranial aneurysms followed by serial MRA. Acta Neurochir (Wien) 155:211-216, 2013

24. Miyazawa N, Akiyama I, Yamagata Z: Risk factors for growth of unruptured intracranial aneurysms: follow-up study by serial $0.5-\mathrm{T}$ magnetic resonance angiography. Neurosurgery 58:1047-1053, 2006

25. Morita A, Kirino T, Hashi K, Aoki N, Fukuhara S, Hashimoto N, et al: The natural course of unruptured cerebral aneurysms in a Japanese cohort. N Engl J Med 366:2474-2482, 2012

26. Ronkainen A, Puranen MI, Hernesniemi JA, Vanninen RL, Partanen PL, Saari JT, et al: Intracranial aneurysms: MR angiographic screening in 400 asymptomatic individuals with increased familial risk. Radiology 195:35-40, 1995

27. So TY, Dowling R, Mitchell PJ, Laidlaw J, Yan B: Risk of growth in unruptured intracranial aneurysms: a retrospective analysis. J Clin Neurosci 17:29-33, 2010

28. Sonobe M, Yamazaki T, Yonekura M, Kikuchi H: Small unruptured intracranial aneurysm verification study: SUAVe study, Japan. Stroke 41:1969-1977, 2010

29. Sprengers ME, Schaafsma JD, van Rooij WJ, van den Berg R, Rinkel GJ, Akkerman EM, et al: Evaluation of the occlusion status of coiled intracranial aneurysms with MR angiography at 3T: is contrast enhancement necessary? AJNR Am J Neuroradiol 30:1665-1671, 2009

30. Tsutsumi K, Ueki K, Morita A, Usui M, Kirino T: Risk of aneurysm recurrence in patients with clipped cerebral aneurysms: results of long-term follow-up angiography. Stroke 32:1191-1194, 2001

31. Villablanca JP, Duckwiler GR, Jahan R, Tateshima S, 
Martin NA, Frazee J, et al: Natural history of asymptomatic unruptured cerebral aneurysms evaluated at CT angiography: growth and rupture incidence and correlation with epidemiologic risk factors. Radiology 269:258-265, 2013

32. Wermer MJ, van der Schaaf IC, Velthuis BK, Algra A, Buskens E, Rinkel GJ: Follow-up screening after subarachnoid haemorrhage: frequency and determinants of new aneurysms and enlargement of existing aneurysms. Brain 128:2421-2429, 2005

33. Wermer MJ, van der Schaaf IC, Velthuis BK, Majoie CB, Albrecht KW, Rinkel GJ: Yield of short-term follow-up CT/ MR angiography for small aneurysms detected at screening. Stroke 37:414-418, 2006

34. Wiebers DO, Whisnant JP, Huston J III, Meissner I, Brown RD Jr, Piepgras DG, et al: Unruptured intracranial aneurysms: natural history, clinical outcome, and risks of surgical and endovascular treatment. Lancet 362:103-110, 2003

35. Yonekura M: Small unruptured aneurysm verification (SUAVe Study, Japan)-interim report. Neurol Med Chir (Tokyo) 44:213-214, 2004

36. Zipfel GJ, Dacey RG: Update on the management of unruptured intracranial aneurysms. Neurosurg Focus 17(5):E2, 2004

\section{Disclosures}

The authors report no conflict of interest concerning the materials or methods used in this study or the findings specified in this paper.

\section{Author Contributions}

Conception and design: Serrone, Tackla, Grossman, Ringer. Acquisition of data: Serrone, Tackla, Gozal, Gogela, Vuong, Kosty, Steiner, Krueger. Analysis and interpretation of data: Serrone, Gozal, Gogela, Grossman. Drafting the article: Serrone, Gozal, Hanseman, Grossman, Ringer. Critically revising the article: Serrone, Tackla, Gozal, Gogela, Grossman, Ringer. Reviewed submitted version of manuscript: Serrone, Tackla, Gozal, Hanseman, Vuong, Kosty, Steiner, Krueger, Grossman, Ringer. Approved the final version of the manuscript on behalf of all authors: Serrone. Statistical analysis: Serrone, Gozal, Hanseman. Administrative/technical/material support: Ringer. Study supervision: Serrone, Ringer.

\section{Correspondence}

Joseph C. Serrone, Department of Neurosurgery at the UC College of Medicine, 260 Stetson St., 2200, P.O. Box 670515, Cincinnati, OH 45267-0515. email: jserrone@gmail.com. 